\title{
Changes in physical-mechanical properties and structure of ferritic-pearlitic steel 15Kh2NMFA caused by severe low-temperature deformation and exposure to alternating magnetic field
}

\author{
V.I. Sokolenko, A.V. Mats, V.I. Karas', V.S. Okovit, N.A. Chernyak, and V.M. Gorbatenko \\ National Science Center "Kharkov Institute of Physics and Technology" \\ 1 Akademicheskaya Str., Kharkov 61108, Ukraine \\ E-mail: vsokol@kipt.kharkov.ua
}

Received October 30, 2014, published online February 23, 2015

\begin{abstract}
It is shown for the low-plasticity ferritic-pearlitic steel 15Kh2NMFA (2Cr, $1 \mathrm{Ni}, 0.5 \mathrm{Mn}, 0.5 \mathrm{Mo})$, subjected to severe rolling deformation at $90 \mathrm{~K}$, that the treatment by the alternating magnetic field results in a substantial decrease of the yield strength and an increase of fracture stress, total elongation, necking and dynamic shear modulus. This is accompanied by reduction of the internal friction background and coercive force. The changes in the physical-mechanical properties and the structure are related to magnetic- and electrical-nature processes giving rise to stress relaxation in the microvolumes with a high density of deformation defects.
\end{abstract}

PACS: $75.50 . \mathrm{Bb}$ Fe and its alloys;

81.40.-Z Treatment of materials and its effects on microstructure, nanostructure, and properties;

81.70.Bt Mechanical testing, impact tests, static and dynamic loads;

83.85.St Stress relaxation.

Keywords: low-temperature deformation, ferritic-pearlitic steel, magnetic treatment, structure, physicalmechanical properties.

\section{Introduction}

The discovery of the magnetoplastic effect (MPE) [1,2] and electroplastic effect $[3,4]$ has stimulated extensive studies of the influence of constant and alternating magnetic fields on the structure and mechanical properties of ferromagnetics and antiferromagnetics [2], and also, of ionic and covalent crystals that have no magnetic order [5]. The MPE was studied under conditions of active deformation and creep. The observed effects were associated with changes in both the dynamics of dislocations in their interaction with the spin subsystem, and overcomeable barrier characteristics.

The effect of a magnetic field on the structure and physical-mechanical properties of magnetic materials as well as weld joints subjected previously to different types of action (deformation, thermal, radiation, etc.) is of fundamental and applied interest.

Thus, a significant increase in the impact toughness and temperature of ductile-brittle transition was observed for the weld joint of the ferritic-pearlitic vessel steel 15Kh2NMFA ( $2 \mathrm{Cr}, 1 \mathrm{Ni}, 0.5 \mathrm{Mn}, 0.5 \mathrm{Mo}$ ) because of the magnetic field action [6]. For the strained samples of the steel a strong decrease in microhardness as a result of this effect was observed [7].
The aim of the present work was to investigate the changes in the structure, mechanical, dissipative and magnetic characteristics of severely deformed steel 15Kh2NMFA, resulting from exposure to an alternating magnetic field.

\section{Material and research methods}

Several thin ( $h \sim 1 \mathrm{~mm}$ ) plates were cut from a massive billet of ferritic-pearlitic steel 15Kh2NMFA after standard factory thermomechanical treatment. Part of them was deformed by rolling at $T=90 \mathrm{~K}$ up to $57 \%$. The samples for mechanical testing with the length of the working part $L=15 \mathrm{~mm}$ and cross-sectional areas $S_{1}=2 \cdot 0.7 \mathrm{~mm}^{2}$ and $S_{2}=2.0 .3 \mathrm{~mm}^{2}$ were prepared from initial and strained plates, respectively, and were characterized by resistivity of $\sim 3.1 \cdot 10^{-5} \mathrm{Ohm} \cdot \mathrm{cm}$ and $\sim 3.2 \cdot 10^{-5} \mathrm{Ohm} \cdot \mathrm{cm}$ at room temperature.

Mechanical characteristics of the samples were determined in the temperature range of 138-293 K under uniaxial tension at a rate of $10^{-3} \mathrm{~s}$. The tests at $T<293 \mathrm{~K}$ were performed in the environment of cooled gasoline with an accuracy of $0.5 \mathrm{~K}$. The magnetic field ( $f=50 \mathrm{~Hz}$ ) was generated by a solenoid calibrated with a Hall sensor. 
To exclude from consideration the effects of Joule heating during magnetic treatment, the samples were cooled with running water. By varying the magnetic field strength in the range of 50-1000 Oe and the exposure duration we have determined the optimal mode $H=500$ Oe, $t=15 \mathrm{~min}$ for providing the maximum effect in the change of mechanical properties of the deformed samples.

Field dependences of magnetization $M(H)$ were measured at $T=300 \mathrm{~K}$ using a special magnetometer KRM-Z-MA. In the measurements, the broad face of the sample was oriented perpendicular to the magnetic field direction. Measurements of the magnetic characteristics were performed on the same sample before and after the magnetic treatment. From the measured $M(H)$ we have calculated the value of the coercive force $H_{c}$.

The internal friction $\delta$ and the shear modulus $G$ of the samples in different structural states were investigated using the technique of reverse torsion pendulum at a frequency of $\sim 0.5 \mathrm{~Hz}$ in the temperature range of 300-800 K.

\section{Results and discussion}

\subsection{Mechanical characteristics}

Table 1 gives the characteristics of strength $\left(\sigma_{02}\right.$ is the yield strength, $\sigma_{B}$ is the ultimate tensile strength, $\sigma_{f}$ is the fracture stress) and plasticity ( $\varepsilon_{u}$ is the uniform elongation, $\varepsilon_{\text {tot }}$ is the total elongation, $\psi$ is the necking) for steel 15Kh2NMFA in the initial state in the temperature range of 293-138 K.

At $T=293 \mathrm{~K}$, the $\sigma_{02}, \sigma_{B}, \varepsilon_{0}$, and $\psi$ values are in good agreement with the characteristics of the steel according to the technical specification [8]. With lowering of temperature the steel hardens; this is accompanied by decrease in plasticity. As can be seen from Table 1, as the temperature decreases from $218 \mathrm{~K}$ down to $138 \mathrm{~K}$, the $\sigma_{f}$ value remains practically unchanged, but the necking is markedly reduced.

A sharp increase in the yield strength at temperatures below (0.15-0.2) $T_{m}$ ( $T_{m}$ is the melting temperature) is typical for bcc transition metals and alloys. This accounts for a change in the character of fracture from ductile to brittle that occurs in a wide range of temperatures (e.g., see Ref. 9).

At tensile test conditions, the upper boundary of the ductile-brittle transition $T_{b}^{h}$ is determined by the onset of fracture stress $\sigma_{f}$ decrease against the background of some

Table 1. Mechanical characteristics of steel 15Kh2MNFA in the initial state

\begin{tabular}{c|c|c|c|c|c|c}
\hline \hline \multirow{2}{*}{$T, \kappa$} & $\sigma_{02}$ & $\sigma_{B}$ & $\sigma_{f}$ & $\varepsilon_{u}$ & $\varepsilon_{\text {tot }}$ & $\psi$ \\
\cline { 2 - 7 } & \multicolumn{3}{|c}{$\mathrm{MPa}$} & \multicolumn{3}{|c}{$\%$} \\
\hline \hline 293 & 500 & 620 & 1140 & 7 & 17 & 60 \\
273 & 500 & 660 & 1230 & 7 & 16 & 60 \\
258 & 580 & 730 & 1700 & 6 & 10 & 70 \\
218 & 590 & 730 & 2060 & 6 & 10 & 71 \\
138 & 700 & 900 & 2060 & 6 & 11 & 58 \\
\hline \hline
\end{tabular}

decrease in necking $\psi$. The fractographic manifestation of this boundary is represented by the signs of cleavage on the fractured surface. Taken the above mentioned into consideration, we can suppose that for the vessel steel in the initial state, the $T_{b}^{h}$ value should lie in the temperature range of 218-138 K. Considering that with the test temperature decrease from 218 to $138 \mathrm{~K}$, the $\sigma_{f}$ value remains almost unchanged, but the $\psi$ value decreases by $~ 18 \%$, the upper boundary of the brittle-ductile transition can be estimated from the lower value of temperature in the range indicated above, i.e. $T=138 \mathrm{~K}\left(-135^{\circ} \mathrm{C}\right)$.

It should be noted that the values of ductile-brittle transition temperature $T_{x 0}$ determined under conditions of uniaxial tension, are lower than the values obtained from toughness tests. The last-mentioned values for the steel 15Kh2NMFA vary significantly among different authors. According to [10], for the "secondary sorbite" state, the $T_{x 0}$ value equals $80{ }^{\circ} \mathrm{C}$ for small-size samples, and $-20^{\circ} \mathrm{C}$ for standard samples. The $T_{x 0}=-129^{\circ} \mathrm{C}$ was obtained in [11] for small Sharpie specimens in the state of "tempering bainite".

The mechanical characteristics of the deformed steel are given in Table 2. From a comparison between the data in Tables 1 and 2 it is seen that in response to severe lowtemperature deformation, typical increase of strength and decrease of plasticity take place. As for the upper boundary of the ductile-brittle transition of the steel in deformed state, here the reduction of $\psi$ to the same value (17-18\%) as in the initial state, occurs in the range of 168-138 K. Considering that the tendency to the decrease of fracture stress is observed at $168 \mathrm{~K}$, which is accompanied by decrease of $\psi$, this value of temperature can be regarded as an upper boundary of ductile-brittle transition for the deformed state. Thus, as a result of severe low-temperature deformation, there is a noticeable increase (at least by $30 \mathrm{~K}$ ) in the beginning of the ductile-brittle transition.

As it can be seen from the comparison of the data given in Tables 2 and 3 the deformed samples after magnetic field exposure are characterized by substantially lower values of yield strength and higher $\sigma_{f}$ values over the entire temperature range. In particular, $\sigma_{02}$ decreases from $810 \mathrm{MPa}$ down to $660 \mathrm{MPa}, \sigma_{f}$ increases from $1590 \mathrm{MPa}$ up to $1700 \mathrm{MPa}, \psi$ increases by $20 \%$ at $T=293 \mathrm{~K}$. Note

Table 2. Mechanical characteristics of steel 15Kh2MNFA in deformed state

\begin{tabular}{c|c|c|c|c|c|c}
\hline \hline \multirow{2}{*}{$T, \mathrm{~K}$} & $\sigma_{02}$ & $\sigma_{B}$ & $\sigma_{f}$ & $\varepsilon_{u}$ & $\varepsilon_{\text {tot }}$ & $\psi$ \\
\cline { 2 - 7 } & \multicolumn{3}{|c|}{$\mathrm{MPa}$} & \multicolumn{3}{|c}{$\%$} \\
\hline \hline 293 & 810 & 910 & 1590 & 1.2 & 2.2 & 49 \\
273 & 820 & 900 & 1200 & 1.0 & 2.0 & 40 \\
258 & 880 & 950 & 1730 & 0.8 & 1.5 & 50 \\
218 & 970 & 980 & 1920 & 0.6 & 1.7 & 54 \\
198 & 980 & 1060 & 2100 & 0.5 & 1.7 & 54 \\
168 & 1050 & 1070 & 2300 & 0.5 & 1.8 & 45 \\
138 & 1080 & 1090 & 2280 & 0.5 & 1.8 & 39 \\
\hline \hline
\end{tabular}


Table 3. Mechanical characteristics of the deformed steel 15Kh2MNFA after exposure to the alternating magnetic field $H=$ $=500 \mathrm{Oe}, t=15 \mathrm{~min}$

\begin{tabular}{c|c|c|c|c|c|c}
\hline \hline \multirow{2}{*}{$T, \mathrm{~K}$} & $\sigma_{02}$ & $\sigma_{B}$ & $\sigma_{f}$ & $\varepsilon_{u}$ & $\varepsilon_{\text {tot }}$ & $\psi$ \\
\cline { 2 - 7 } & \multicolumn{3}{|c|}{$\mathrm{MPa}$} & \multicolumn{3}{|c}{$\%$} \\
\hline \hline 293 & 660 & 790 & 1700 & 0.7 & 2.4 & 58 \\
273 & 670 & 800 & 1290 & 1.3 & 2.6 & 43 \\
258 & 800 & 920 & 1880 & 0.8 & 2.0 & 63 \\
218 & 890 & 1000 & 2020 & 1.2 & 1.8 & 60 \\
198 & 1000 & 1110 & 2300 & 1.2 & 2.5 & 66 \\
168 & 1000 & 1150 & 2760 & 1.3 & 2.5 & 60 \\
138 & 950 & 1150 & 2620 & 1.5 & 3.0 & 64 \\
\hline \hline
\end{tabular}

that the fracture of the specimen after magnetic treatment proceeds with the formation of the cone and cup ( $\psi \approx 58 \%)$, while the fracture of the specimen without magnetic treatment occurs by cutting ( $\psi \approx 49 \%$ ) (see Fig. 1 ).

The data in Table 3 demonstrate the changes in the mechanical properties of the deformed vessel steel as a result of magnetic treatment. Even at low temperatures, the samples exposed to the magnetic field are getting deformed, showing hardening and a noticeable reserve of plasticity. So, the total and uniform elongations at $T=138 \mathrm{~K}$ amount, respectively, to 3.0 and $1.5 \%$ versus 1.8 and $0.5 \%$ for the strained state.

\subsection{Internal friction and shear modulus}

There are no anomalies of $\delta(T)$ and $G(T)$ in the temperature range investigated for the material in the initial state (Figs. 2, 3; curve 1 ). At $T=270 \mathrm{~K}$, the background level of $\delta \approx 1.5 \cdot 10^{-3}$. In the range of $600-700 \mathrm{~K}$, we observed an insignificant monotonic increase in the background, and the maximum $\delta$ value did not exceed $4 \cdot 10^{-3}$. At $T>740 \mathrm{~K}$, the internal friction increases exponentially, that can be the manifestation of the low-temperature branch of the peak located at $T>800 \mathrm{~K}$. The shear modu-

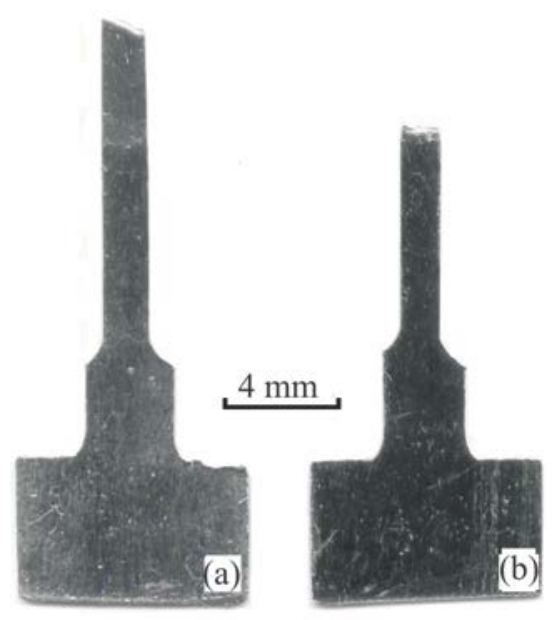

Fig. 1. Fracture mode of the sample before (a) and after exposure to the alternating magnetic field $H=500 \mathrm{Oe}, t=15 \mathrm{~min}$ (b).

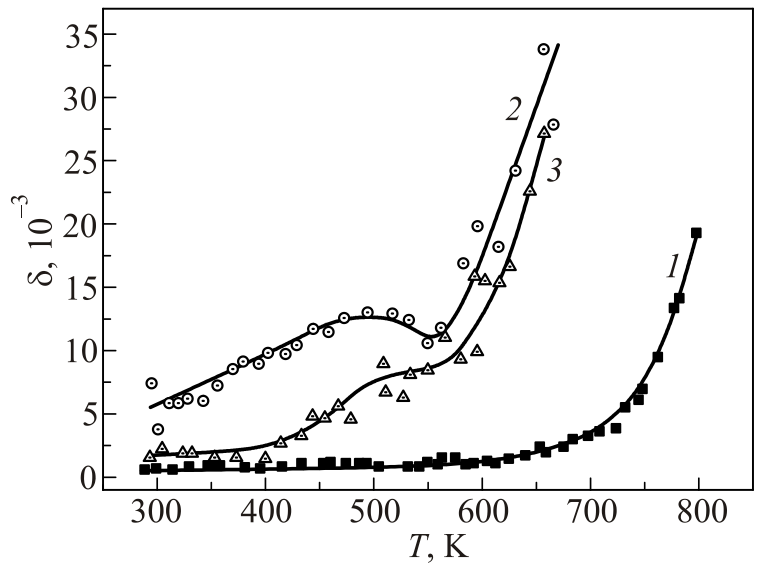

Fig. 2. Temperature dependence of internal friction $\delta$ of steel 15Kh2MNFA in different states: initial (1), after rolling deformation at $90 \mathrm{~K}$ by $57 \%$ (2), state 2 after exposure to the alternating magnetic field $H=500$ Oe, $t=15 \mathrm{~min}$ (3).

lus amounts to $8 \cdot 10^{4} \mathrm{MPa}$ at $T=270 \mathrm{~K}$ and decreases by a factor of $\sim 2.6 \cdot 10^{-3} \mathrm{~K}^{-1}$ as the temperature increases.

The internal friction background increases markedly for the strained state. In the region of $350-570 \mathrm{~K}$, we have observed a broad maximum $\delta$, the right branch of which goes to the line of exponential growth of $\delta$ (Fig. 2, curve 2). The shear modulus is reduced by deformation, in particular, by $\sim 2000 \mathrm{MPa}$ at $T=290 \mathrm{~K}$. With increasing temperature above $500 \mathrm{~K}$, the strain effect is difficult to identify (Fig. 3; curves 1, 2).

After processing by alternating magnetic field, the shear modulus values of the deformed samples remain close to those in the initial state (Fig. 3, curve 3). The background internal friction of the processed samples is reduced in the region of 290-420 K (Fig. 2, curve 3). A broad maximum which was identified for the strained state degrades, thereby indicating the occurrence of processes of partial stress relaxation. The sharp ascending branch of $\delta$ is shifted to higher temperatures.

Note that for the material in the initial state, the mentioned magnetic treatment exerted practically no effect on the properties under investigation.

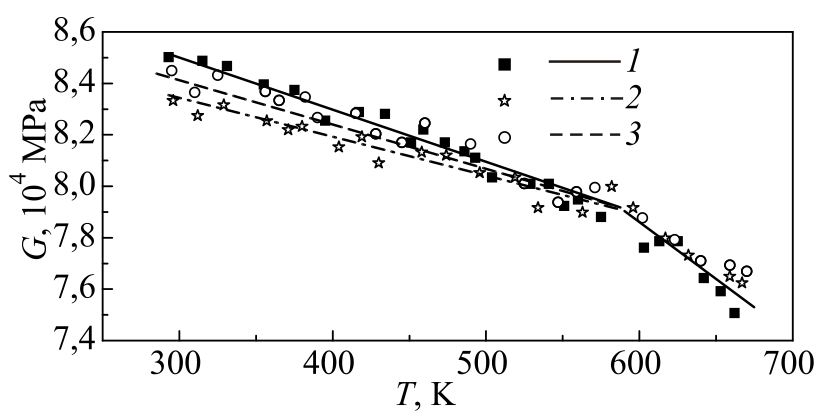

Fig. 3. Temperature dependence of shear modulus of steel 15Kh2MNFA in different states: initial (1), after rolling deformation at $77 \mathrm{~K}$ by $57 \%$ (2), state 2 after exposure to the alternating magnetic field $H=500$ Oe, $t=15 \mathrm{~min}$ (3). 


\subsection{Magnetic characteristics}

It follows from the field dependence of the magnetization $M(H)$ that for the deformed specimens exposed to an alternating magnetic field, the magnetic permeability becomes greater and is accompanied by a decrease in the area of the hysteresis loop, previously increased as a result of deformation. The obtained hysteresis loops are close to the ultimate magnetization curves, taking into account the demagnetization factor. As an example, Table 4 gives the values of coercive force $H_{C}$ of the sample. The decrease in $H_{C}$ reaches $\approx 10 \%$ after magnetic treatment in comparison with $H_{C}$ of the strained state.

Table 4. Coercive force of steel 15Kh2MNFA in different states

\begin{tabular}{c|c}
\hline \hline State & $H_{c}, \mathrm{~A} / \mathrm{m}$ \\
\hline \hline Initial & 920.0 \\
Deformation $\delta=57 \%, T=90 \mathrm{~K}$ & 1820.0 \\
Magnetic treatment & 1690.0 \\
\hline \hline
\end{tabular}

\subsection{Structure characteristics}

In the steel samples a heterogeneous structure is formed as a result of severe low-temperature rolling deformation. The orientation of the structural elements relative to the rolling plane influences the type and amount of the defects.

In the microvolumes, the orientation of which is slightly different from textural, the plastic flow is sufficiently developed and is accompanied by the formation of cellular and fragmented structures having the transverse size of cells of $\approx 0.25 \mu \mathrm{m}$. Misorientations between different fragments or cells come up to $\sim 6^{\circ}$, and the total misorientation reaches $18^{\circ}$ in the microvolume under study (Fig. 4(a)). In the microareas with higher orientation indexes, a strongly strained structure was observed in the form of dense dislocation pileups of the same sign which cause misorientations of up to $19^{\circ}$ (Fig. 4(b)). This testifies about too high elastic distortions of the lattice, and too incomplete realization of rotation modes to establish the texture orientations. The structure also shows the separate high-angle boundaries of disclination origin, with misorientations of $\sim 21^{\circ}$. A high degree of heterogeneity of the defect structure specifies a high level of internal stresses with their local concentration both within the finite-size microregions, and at the boundaries between the areas having different degrees of imperfection. This is evidenced by the dislocation charges observed at the boundaries, and also by strong blurring of reflections in the electron diffraction patterns.

We have noted the following structural changes resulting from exposure to an alternating magnetic field. In the areas with developed dislocation structure, i.e., cellular ensembles, the number of dislocation boundaries increased with a decrease in the average cell size down to $\approx 0.17 \mu \mathrm{m}$ and their misorientation angles down to $5^{\circ}$ (Fig. 5(a)).

The effects of plastic relaxation can be seen more clearly in the microvolumes with higher level of elastic stresses. In the areas with local inhomogeneities, the internal stress concentration is reduced due to dislocation multiplication and redistribution, and also due to dispersal of dislocations directly in the pileups. At the same time, the disorientation angles are reduced to $9^{\circ}$. We observed the formation of boundaries at the sites of powerful piling-up of the sametype name dislocations (Fig. 5(b)), and it can also be interpreted as aspects of relaxation.

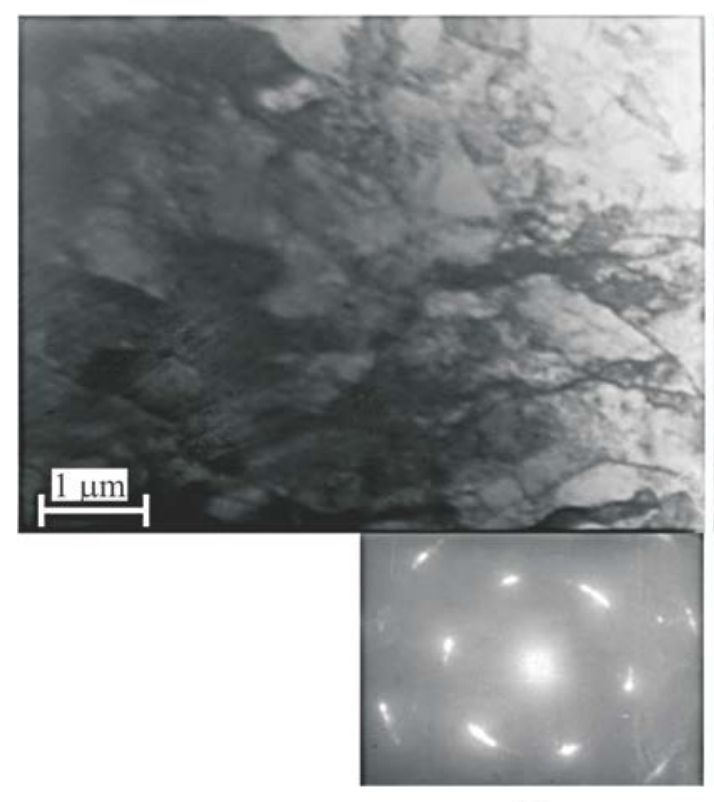

(a)

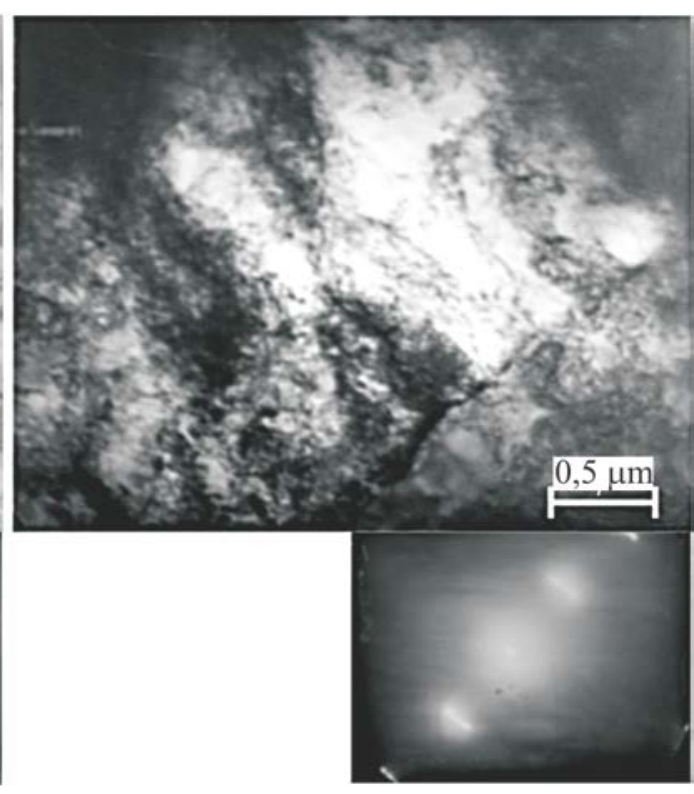

(b)

Fig. 4. Structure of steel 15Kh2MNFA after rolling deformation by $57 \%$ at $90 \mathrm{~K}$ (see text). 


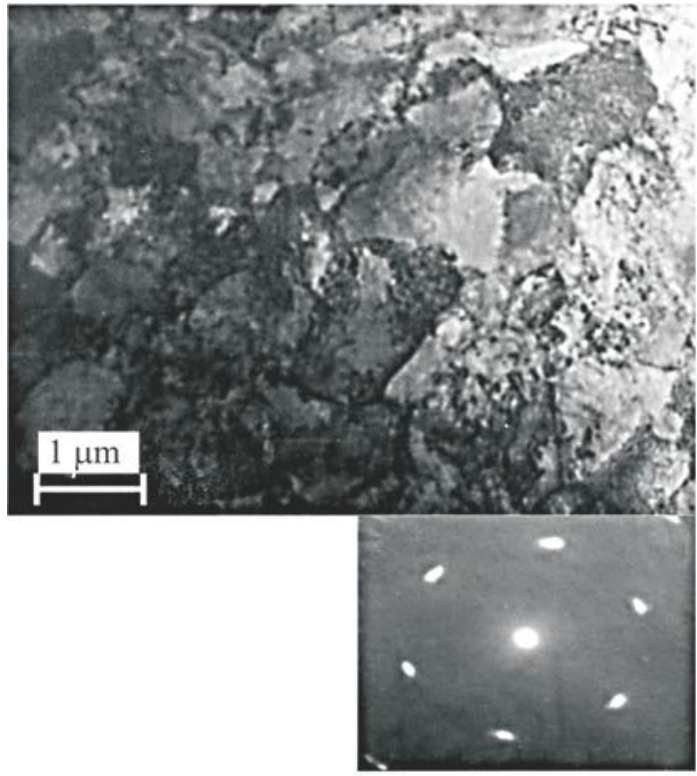

(a)

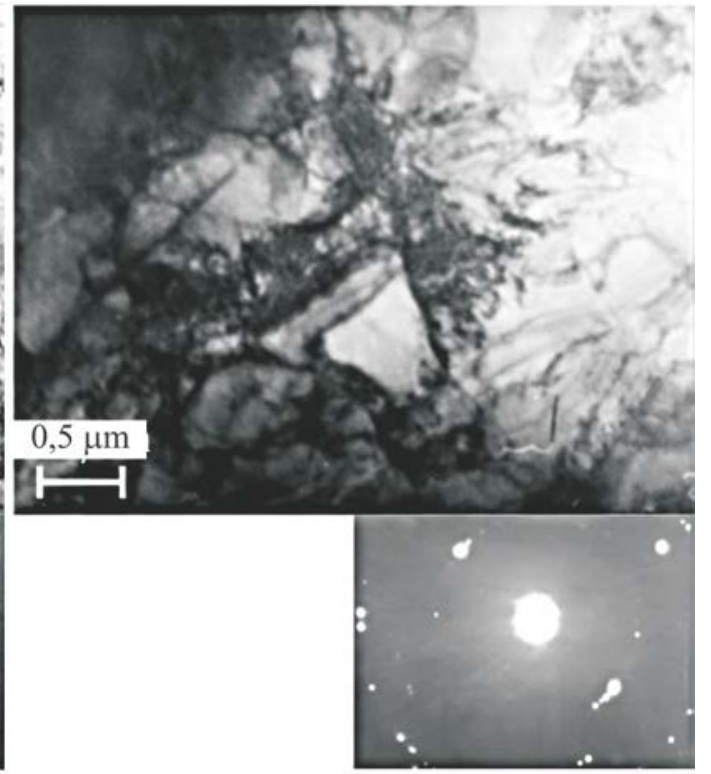

(b)

Fig. 5. Structure of steel $15 \mathrm{Kh} 2 \mathrm{MNFA}$ after rolling deformation by $57 \%$ at $90 \mathrm{~K}$ and exposure to the alternating magnetic field $H=500$ Oe, $t=15 \mathrm{~min}$ (see text).

Let us briefly consider possible channels for dissipation of energy of an alternating magnetic field, which causes relaxation processes in microvolumes of the ferromagnetic material with a high internal-stress level.

Relaxation processes can take place in dislocation pileups of high power and at the boundaries of fragments during their interaction with the boundaries of the magnetic domains moving under the influence of an external variable magnetic field.

Figure 6 shows the scheme illustrating possible decrease of local internal stresses $(\tau)$ due to reduction in the number of dislocations in the inhibited pileups owing to passing the domain boundary. The displacement of dislocations to the neighboring slip planes is possible due to the effects of nonlinear dynamics of dislocations in consequence of genera- tion and motion of double kinks under the action of alternating magnetostrictive stresses of small amplitude [12]. In addition, magnetostrictive deformations, including domain wall displacement and rotation of the magnetization vector, cause effective softening of the elastic moduli [13], that facilitates dislocation migration.

The magnetic relaxation of the orientation aftereffect type [14] will promote the displacement of interstitial impurities and other defects, characterized by local magnetic moments. As a result, one would expect depinning dislocations, redistribution and runoff of defects to the interfaces and other advantageous sinks that will result in a lower flow stress. The additional channel of stress relaxation can be realized due to the action of unpinned dislocation sources.

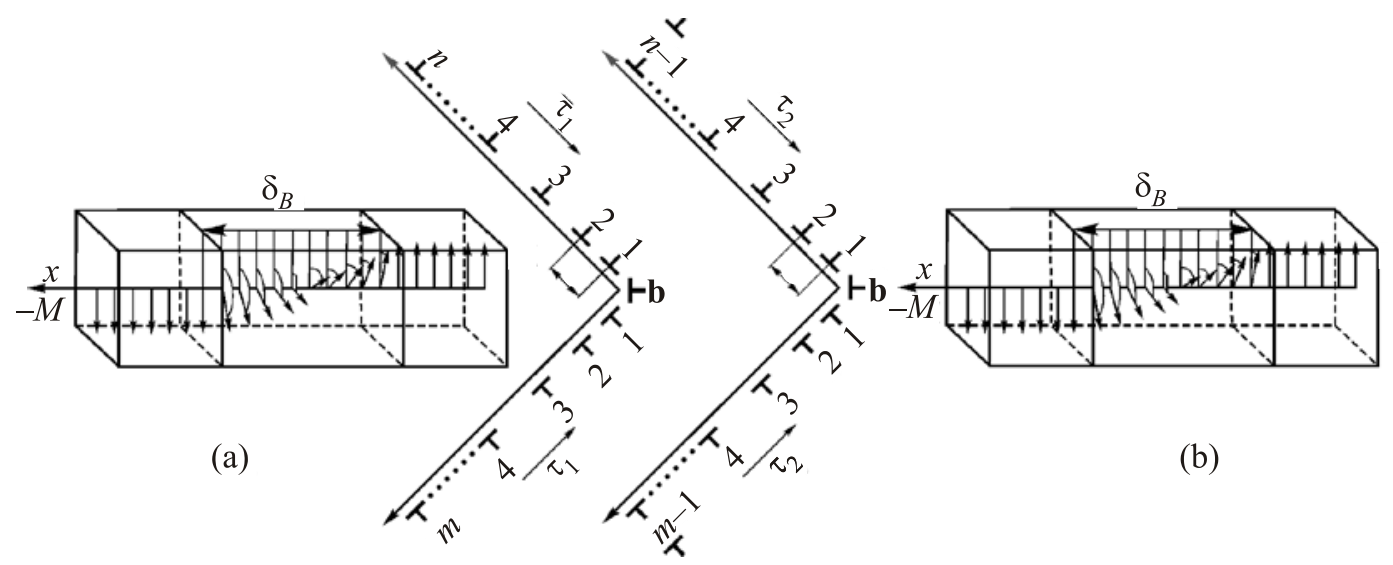

Fig. 6. Decrease in the number of dislocations in the plane pileup (according to the model of Cottrell) when passing the domain boundary through the pileup (left to right), which corresponds to $\tau_{2}<\tau_{1}$. Arrows in different positions show spin rotation in the Bloch wall of width $\delta_{B}$ (see text). 
The alternating magnetic field, apart from the magnetic component, generates in the volume of the conductor an electric field that acts on the electron subsystem. Lately, the understanding of the mechanisms of the electron subsystem excitation effect on real crystalline solids has received considerable attention [15]. The essence is that under the influence of the electric field there appear high-energy electrons, which interact with phonons, thereby generating many phonons of energies close to the Debye energy, which interact with dislocations, detach them from point defects (stoppers) $[16,17]$. The separation of the contributions of magnetic and electric components of the alternating magnetic field at its impact on the real crystal defect structures calls for consideration of microscopic models.

\section{Conclusions}

1. For the ferritic-pearlitic steel 15Kh2NMFA (2Cr, $1 \mathrm{Ni}, 0.5 \mathrm{Mn}, 0.5 \mathrm{Mo}$ ) subjected to severe low-temperature deformation and characterized by low plasticity and high strength it has been shown, that the treatment by the alternating magnetic field results in a substantial decrease of yield strength (18\%) and increase of fracture stress (up to $10 \%$ ), total elongation (up to $65 \%$ ) and necking (up to $60 \%$ ) in the temperature range of $293-138 \mathrm{~K}$. These effects were accompanied by a decrease in the internal friction background by a factor $\sim 2$ and the coercive force (up to $\approx 10 \%$ ), and a growth of the dynamic shear modulus in the range of 300-500 K.

2. The changes in the physical-mechanical properties and structure are related to the magnetic- and electricnature processes that give rise to stress relaxation in the microvolumes with a high level of internal stresses (highangle boundaries, dense dislocation pileups).

This work was in part carried out with the support of the National Academy of Sciences of Ukraine (Project 61-02-14(U)) and the Russian Fund of Fundamental Research (Project 14-02-90248(R)).

1. S. Hayashi, S. Takahashi, and M. Yamamoto, J. Phys. Soc. Jpn. 25, 381 (1968).

2. I.M. Neklyudov, Ya.D. Starodubov, and V.I. Sokolenko, Ukr. J. Phys. 50, A113 (2005) (in Russian).
3. O.A. Troitskii and V.I. Likhtman, Dokl. Acad. Nauk SSSR 148, 332 (1963) (in Russian).

4. V.E. Gromov, B.Ya. Tsellermaier, and V.I. Bazaikin, Elektrostimulirovannoe Volochenie: Analiz Protsessa i Mikrostruktura, Nedra, Moskow (1996) (in Russian).

5. R.B. Morgunov, Phys. Usp. 47, 125 (2004) (in Russian).

6. I.M. Neklyudov, V.M. Azhazha, K.A. Yushchenko, V.I. Sokolenko, A.V. Mats, V.M. Netesov, and V.V. Varganov, Fizika i Khimiya Obrabotki Materialov No. 1, 84 (2011) (in Russian).

7. I.M. Neklyudov, V.M. Azhazha, V.I. Sokolenko et al. Proc. 18th Int. Conf. Phys. Rad. Phenom. \& Rad. Mat. Sci. Kharkov, NSC KIPT, 2008, p. 156 (in Russian).

8. Technical Regalement 08 93-013-00212179-2003 (Instead Technical Regalement 198.765-78), 2003 (in Russian).

9. V.I. Trefilov, V.F. Moiseev, E.V. Pechkovsky et al. Deformation Hardening and Fracture of Polycrystalline Metals, Naukova Dumka, Kiev (1989) (in Russian).

10. E.A. Krainiuk, V.G. Lesnaya, L.S. Ozhigov, and V.I. Savchenko, Problems of Atomic Science and Technology. Ser.: Phys. Rad. Damages \& Rad. Mat. Sci. 85, No. 3, 137 (2004) (in Russian).

11. B.A. Gurovich, E.A. Kuleshova, C.V. Fedorova et al., Proc. 7th Int. Sci. Tech. Conf. "Ensuring the Safety of NPP with VVER”, ACB “Gidropress”, Podolsk, Russia, May 17-20 (2011) (in Russian).

12. I.M. Neklyudov, V.I. Sokolenko, and V.I. Tkachenko, Mat. 9th Int. Conf. "Physical Phenomena in Solids", Kharkov, December 1-4 (2009), p. 93 (in Russian).

13. A. Sosin and D.V. Kifer, Radiation Exposure on the Internal Friction, Elastic Modulus and the Magnetic Aftereffect in Metals, in: Microplasticity. Metallurgiya, Moscow (1972) (in Russian).

14. A.I. Mitsek and V.N. Pushkar, Real Crystals with Magnetic Order, Naukova Dumka, Kiev (1978) (in Russian).

15. V.I. Dubinko, V.I. Karas', V.F. Klepikov, P.N. Ostapchuk, and I.F. Potapenko, Problems of Atomic Science and Technology. Ser.: Phys. Rad. Damages \& Rad. Mat. Sci. 94 \#2, 158 (2009) (in Russian).

16. V.E. Zakharov and V.I. Karas', Usp. Fiz. Nauk 56, 49 (2013).

17. V.I. Karas', A.M. Vlasenko, V.N. Voyevodin, and V.I. Sokolenko, East Europe J. Phys. 1, 306 (2014). 